\title{
Inpatient Management of Diabetic Foot Infections: A Review of the Guidelines for Hospitalists
}

\author{
Emilia G. Thurber, BA¹, Flora Kisuule, $\mathrm{MD}^{2}$, Casey Humbyrd, $\mathrm{MD}^{3}$, Jennifer Townsend, $\mathrm{MD}^{4 *}$
}

${ }^{1}$ Johns Hopkins University School of Medicine, Baltimore, Maryland; '2Division of Hospital Medicine, Johns Hopkins University School of Medicine, Baltimore, Maryland; ${ }^{3}$ Department of Orthopaedic Surgery, Johns Hopkins University School of Medicine, Baltimore, Maryland; ${ }^{4}$ Division of Infectious Diseases, Johns Hopkins University School of Medicine, Baltimore, Maryland.

Diabetic foot infections (DFIs) are common and represent the leading cause for hospitalization among diabetic complications. Without proper management, DFls may lead to amputation, which is associated with a decreased quality of life and increased mortality. However, there is currently significant variation in the management of DFls, and many providers fail to perform critical prevention and assessment measures. In this review, we will provide an overview of the diagnosis, management, and discharge planning of hospitalized patients with DFls to guide hospitalists in the optimal inpatient care of patients with this condition. Journal of Hospital Medicine 2017;12:994-1000. Published online first September 20, 2017. (C) 2017 Society of Hospital Medicine
Diabetic foot infection (DFI) is a common result of diabetes and represents the most frequent complication requiring hospitalization and lower extremity amputation. ${ }^{1,2}$ Hospital discharges related to diabetic lower extremity ulcers increased from 72,000 in 1988 to 113,000 in $2007,{ }^{3}$ and admissions related to infection rose 30\% between 2005 and 2010. ${ }^{2}$ Ulceration and amputation are associated with a $40 \%$ to $50 \%$ 5-year mortality rate. ${ }^{4,5}$

Aggressive risk-factor management and interprofessional care can significantly reduce major amputations and mortality. ${ }^{6-13}$ Consistent and high-quality care for patients admitted with DFI is essential for optimizing outcomes; however, management varies widely, and critical assessment and prevention measures are often not employed by providers. ${ }^{14}$ This review synthesizes recommendations from existing guidelines to provide an overview of the best practices for the diagnosis, management, and discharge of DFI in the hospital setting (Supplementary Table 1, Supplementary Figure).

\section{DETECTION AND STAGING OF INFECTION}

The first step in the management of a DFI is a careful assessment of the presence and depth of infection. ${ }^{15}$ The Infectious Diseases Society of America (IDSA) guidelines recommend using at least 2 signs of classic inflammation (erythema, warmth, swelling, tenderness, or pain) or purulent drainage to diagnose soft tissue infection. ${ }^{1,15,16}$ Patients with ischemia may present atypically, with nonpurulent secre-

\footnotetext{
*Address for correspondence and reprint requests: Jennifer Townsend, MD, Division of Infectious Diseases, Johns Hopkins Bayview Medical Center, 5200 Eastern Ave, MFL Center Tower \#381, Baltimore, MD 21224; Telephone: 410550-9080; Fax: 410-550-1169; E-mail: jholme27@jhmi.edu
}

Additional Supporting Information may be found in the online version of this article.

Received: January 16, 2017; Revised: April 26, 2017;

Accepted: April 21, 2017

2017 Society of Hospital Medicine DOI 10.12788/jhm.2842 tions, friable or discolored granulation tissue, undermining of wound edges, and foul odor. ${ }^{1,15,16}$ Additional risk factors for DFI include ulceration for more than 30 days, recurrent foot ulcers, a traumatic foot wound, severe peripheral arterial disease (PAD) in the affected limb (ankle brachial index $[\mathrm{ABI}]<0.4)$, prior lower extremity amputation, loss of protective sensation, end-stage renal disease, and a history of walking barefoot. ${ }^{15,17,18}$

Appropriate classification of wound severity is critical in determining the need for hospitalization, antibiotic selection, surgical intervention, and prognosis. Multiple staging systems that incorporate physical examination findings, markers of systemic inflammation, and ischemia ${ }^{15,19,20}$ have been proposed. The Perfusion, Extent, Depth, Infection, and Sensation (PEDIS) grade was developed as a research tool and incorporates infection, ischemia, neuropathy, wound size, and systemic inflammation. ${ }^{15}$ The International Working Group on the Diabetic Foot (IWGDF) and the IDSA recommend use of the full or simplified PEDIS score in clinical practice (the IWGDF/IDSA Classification, Table 1) because these classifications predicted hospitalization and lower extremity amputation in prospective studies, with amputation rates of $3 \%$ for uninfected ulcers and up to $70 \%$ for severe infection. ${ }^{1,15}$ Patients with PEDIS grade 4 infections also have an increased mean length of stay compared with patients with grade 3 infections. ${ }^{21,22}$

\section{CRITERIA FOR HOSPITALIZATION}

In practice, the decision to admit is based on clinical and systems-based drivers (Supplementary Table 2). The IDSA and IWGDF guidelines recommend hospitalization for patients with severe (PEDIS grade 4) infection, moderate (PEDIS grade 3) infection with certain complications (eg, severe PAD or lack of home support), an inability to comply with required outpatient treatment, lack of improvement with outpatient therapy, or presence of metabolic or hemodynamic instability. ${ }^{1,15}$ Clinicians must also consider the 


\begin{tabular}{|c|c|c|}
\hline Clinical Manifestation of Infection & PEDIS Grade & IDSA Infection Severity \\
\hline No symptoms or signs of infection & 1 & Uninfected \\
\hline $\begin{array}{l}\text { Infection present, as defined by the presence of at least } 2 \text { of the following items: } \\
\text { Local swelling or induration } \\
\text { Erythema } \\
\text { Local tenderness or pain } \\
\text { Local warmth } \\
\text { Purulent discharge (thick, opaque to white or sanguineous secretion). } \\
\text { Local infection involving only the skin and the subcutaneous tissue (without involvement of deeper tissues and without systemic signs as } \\
\text { described below). If erythema, must be }>0.5 \mathrm{~cm} \text { to } \leq 2 \mathrm{~cm} \text { around the ulcer. Excludes other causes of an inflammatory response of the skin } \\
\text { (eg, trauma, gout, acute Charcot neuro-osteoarthropathy, fracture, thrombosis, venous stasis). }\end{array}$ & 2 & Mild \\
\hline $\begin{array}{l}\text { Local infection (as described above) with erythema }>2 \mathrm{~cm} \text { or involving structures deeper than skin and subcutaneous tissues (eg, abscess, } \\
\text { osteomyelitis, septic arthritis, fasciitis), and no systemic inflammatory response signs (as described below). }\end{array}$ & 3 & Moderate \\
\hline $\begin{array}{l}\text { Local infection (as described above) with the signs of SIRS, as manifested by } \geq 2 \text { of the following: } \\
\text { Temperature }>38^{\circ} \mathrm{C} \text { or }<36^{\circ} \mathrm{C} \\
\text { Heart rate }>90 \text { beats per min } \\
\text { Respiratory rate }>20 \text { breaths per min or PaCO2 }<32 \mathrm{~mm} \mathrm{Hg} \\
\text { White blood cell count }>12,000 \text { or }<4000 \text { cells per } \mu \mathrm{L} \text { or } \geq 10 \% \text { immature (band) forms }\end{array}$ & 4 & Severe \\
\hline
\end{tabular}

need for surgical debridement or complex antibiotic choices due to allergies and comorbidities. Hospitalists may also consider admission in cases in which outpatient follow-up cannot be easily arranged (eg, uninsured patients).

Outpatient management may be appropriate for patients with mild infections who are willing to be reassessed within 72 hours, or sooner if the infection worsens. ${ }^{23}$ For patients with moderate infections (eg, osteomyelitis without systemic signs of infection), access to an outpatient interprofessional DFI care team can potentially decrease the need for admission.

\section{DIAGNOSIS OF OSTEOMYELITIS}

Clinical features that raise suspicion for osteomyelitis include ulceration for at least 6 weeks with appropriate wound care and offloading, wound extension to the bone or joint, exposed bone, ulcers larger than $2 \mathrm{~cm}^{2}$, previous history of a wound, multiple wounds, and appearance of a sausage digit. ${ }^{15}$

The gold standard for diagnosis of osteomyelitis is a bone biopsy with histology. In the absence of histology, physicians rely on physical examination, inflammatory markers, and imaging to make the diagnosis. The presence of visible, chronically exposed bone within a forefoot ulcer is diagnostic. The accuracy of a probe to bone test depends on the pretest probability of osteomyelitis. Sensitivity and specificity range from $60 \%$ to $87 \%$ and from $85 \%$ to $91 \%$, respectively. ${ }^{24}$ For patients with a single forefoot ulcer and PEDIS grade 2 or 3 infection, considering both ulcer depth and serum inflammatory markers (ulcer depth greater than $3 \mathrm{~mm}$, or C-reactive protein greater than $3.2 \mathrm{mg} / \mathrm{dL}$; ulcer depth greater than $3 \mathrm{~mm}$, or erythrocyte sedimentation rate greater than $60 \mathrm{~mm} / \mathrm{h}$ ) increases sensitivity to $100 \%$, although the specificity is relatively low ( $55 \%$ and $60 \%$, respectively). ${ }^{25}$ When the diagnosis remains uncertain by physical examination, imaging is necessary for further evaluation.

\section{ROLE OF IMAGING}

All patients with DFI should have plain radiographs to look for foot deformities, soft tissue gas, foreign bodies, and osteomyelitis. If plain radiographs show classic evidence of osteomyelitis, (ie, cortical erosion, periosteal reaction, mixed lucency, and sclerosis in the absence of neuro-osteoarthropathy), advanced imaging is not necessary. However, these changes may not appear on plain films for up to 1 month after infection onset. ${ }^{15,26}$

The purpose of advanced imaging in the inpatient management of DFI is to detect conditions not obvious by physical examination or by plain radiographs that would alter surgical management (ie, deep abscess or necrotic bone) or antibiotic duration (ie, osteomyelitis or tenosynovitis). ${ }^{15}$ Magnetic resonance imaging (MRI) is the diagnostic modality of choice when the wound does not probe to bone and the diagnosis remains uncertain ${ }^{27}$ due to its accuracy and availability. ${ }^{1,15}$ However, MRI cannot always distinguish between infection and neuro-osteoarthropathy, especially in patients who have infection superimposed on a Charcot foot, have had recent surgical intervention, or have osteosynthesis material at the infection site. ${ }^{24}$ If MRI is contraindicated, guidelines vary on the next recommended test. The IDSA and the Society for Vascular Surgery recommend a labeled white blood cell scan combined with a bone scan, whereas the IWGDF recommends a labeled leukocyte scan, a single photon emission computed tomography (SPECT/ $\mathrm{CT}$ ), or a fluorodeoxyglucose positron emission tomogra- 
phy (FDG PET) scan. ${ }^{1,15,19}$ A recent comparison of a labeled white blood cell SPECT/CT versus MRI (using histology as the gold standard) reported that SPECT/CT had a similar sensitivity ( $89 \%$ versus $87 \%$, respectively) and specificity ( $35 \%$ versus $37 \%$, respectively) to MRI. ${ }^{28}$ In practice, physicians should consider which studies are readily available and confidently interpreted by radiologists at their institution.

\section{ASSESSMENT OF ULCER ETIOLOGY}

After infection is diagnosed and staged, clinicians should determine the underlying derangement in order to prevent recurrence after discharge. Common derangements leading to ulceration in diabetics include PAD, neuropathy, muscular tension, altered foot mechanics, trauma, or a combination of the above. ${ }^{1,15,29-31}$ All patients with DFI should undergo pedal perfusion assessment by an $\mathrm{ABI}$, ankle and pedal Doppler arterial waveforms, and either toe brachial index (TBI) or transcutaneous oxygen pressure. ${ }^{1,15,19}$ In cases of suspected calcification, TBI is a more reliable measure of ischemia compared with the ABI. ${ }^{16,19}$ For patients with signs and symptoms of ischemia and an abnormal $\mathrm{ABI}$ or TBI measurement $(\mathrm{ABI}<0.9$ and $\mathrm{TBI}<0.7)$, a nonurgent consultation with a vascular surgeon is recommended, while patients with severe ischemia $(\mathrm{ABI}<0.4)$ usually require urgent revascularization. . $^{15,32}$

A sensory examination with a Semmes-Weinstein monofilament should be conducted to identify patients with loss of protective sensation who may benefit from offloading devices and custom orthotics. ${ }^{15}$ Foot anatomy and mechanics as well as potential Achilles tendon contractures should be evaluated by a foot specialist such as a podiatrist, orthotist, orthopedist, or vascular surgeon, especially if debridement or amputation is being contemplated.

\section{OBTAINING CULTURES}

After diagnosing the infection clinically, appropriately obtained cultures are essential to guide therapy in all except mild cases with no prior antibiotic exposure or MRSA risk. ${ }^{1,15}$ Guidelines strongly recommend that specimens be obtained by biopsy or curettage from deep tissue at the base of the ulcer after the wound has been cleansed and debrided and prior to initiating antibiotics. ${ }^{1,15,33}$ Aspiration of purulent secretions using a sterile needle and syringe is another acceptable culturing method. ${ }^{15}$ While convenient, swab cultures are prone to both false-positive and false-negative results. ${ }^{34}$ Repeat cultures are only needed for patients who are not responding to treatment or for surveillance of resistant organisms. ${ }^{1}$

In cases of osteomyelitis, bone specimens should be sent for culture and histology either during surgical debridement or a bone biopsy. At the time of debridement, cultures and pathology should be sent from the proximal (clean) bone margin in order to document whether there is residual osteomyelitis postdebridement. ${ }^{35}$ For patients not planned for debridement, a bone biopsy is recommended if the diagnosis of osteomyelitis is unclear, response to empiric therapy is poor, broad-spectrum antibiotics are being considered, or the infection is in the midfoot or hindfoot. ${ }^{1,15,19}$ Results from soft tissue or sinus tract specimens should not be used to guide antibiotic selection in osteomyelitis, as several studies suggest that they do not correlate with bone culture results; one retrospective review found a mere $22.5 \%$ correlation between wound swabs and bone biopsy. ${ }^{1,36} \mathrm{~A} 2$-week antibiotic-free period prior to biopsy is recommended in order to minimize the risk of false-negative results but must be balanced with the risk of worsening infection. ${ }^{1,15}$ If possible, the biopsy should be performed through uninfected tissue under fluoroscopy or CT guidance, with 2 to 3 cores obtained for culture and histology. ${ }^{1,15}$

\section{INTERPROFESSIONAL INPATIENT CARE}

A growing number of health systems have created inpatient and/or outpatient interprofessional diabetic foot care teams, and several studies demonstrated an association between these teams and a reduction in major amputations..$^{711,13}$ The goal of the inpatient team is to rapidly triage patients with moderate to severe infections, expedite surgical interventions and culture collection, establish an effective treatment plan, and ensure adherence postdischarge to optimize outcomes. The common core of most teams includes podiatry, endocrinology, wound care, and vascular surgery, but team composition may vary based on the availability of local specialists with interest and expertise in DFI.9.10,33

The division of consultation between podiatry and orthopedic surgery is highly dependent upon individual practice patterns and hospital structure. In general, forefoot ulcers may be managed by podiatry or orthopedic surgery, while severe Charcot deformities are most often treated by orthopedic surgeons. Wound care nurses are often integral to successful wound healing, collaborating across specialties and serving as a weekly or biweekly point of contact for patients.

Early involvement of Infectious Disease (ID) specialists can be useful for guiding antibiotic choices and facilitating follow-up. ID should be involved with patients who require long-term antibiotic therapy (ie, cases of deep-tissue infection that are not completely amputated or debrided), have failed outpatient or empiric therapy, have antibiotic allergies or drug-resistant pathogens, or are being considered for outpatient parenteral antibiotic therapy.

\section{ANTIBIOTIC THERAPY}

Empiric antibiotic therapy should be based on infection severity and the likely causative agent (Figure). Mild cases are managed with oral agents that target Staphylococcus aureus and Streptococcus species such as cephalexin or clindamycin. ${ }^{1,15}$ Antibiotics for moderate (PEDIS grade 3 ) infections can be oral or parenteral (eg, ampicillin-sulbactam or ertapenem) and should include coverage for the above pathogens in addition to Enterobacteriaciae and anaerobes. ${ }^{1,15}$ Empiric anti-MRSA coverage is optional in mild to moderate infections and should be reserved for patients with known risk factors, such as prior colonization, recent hospitalization, 


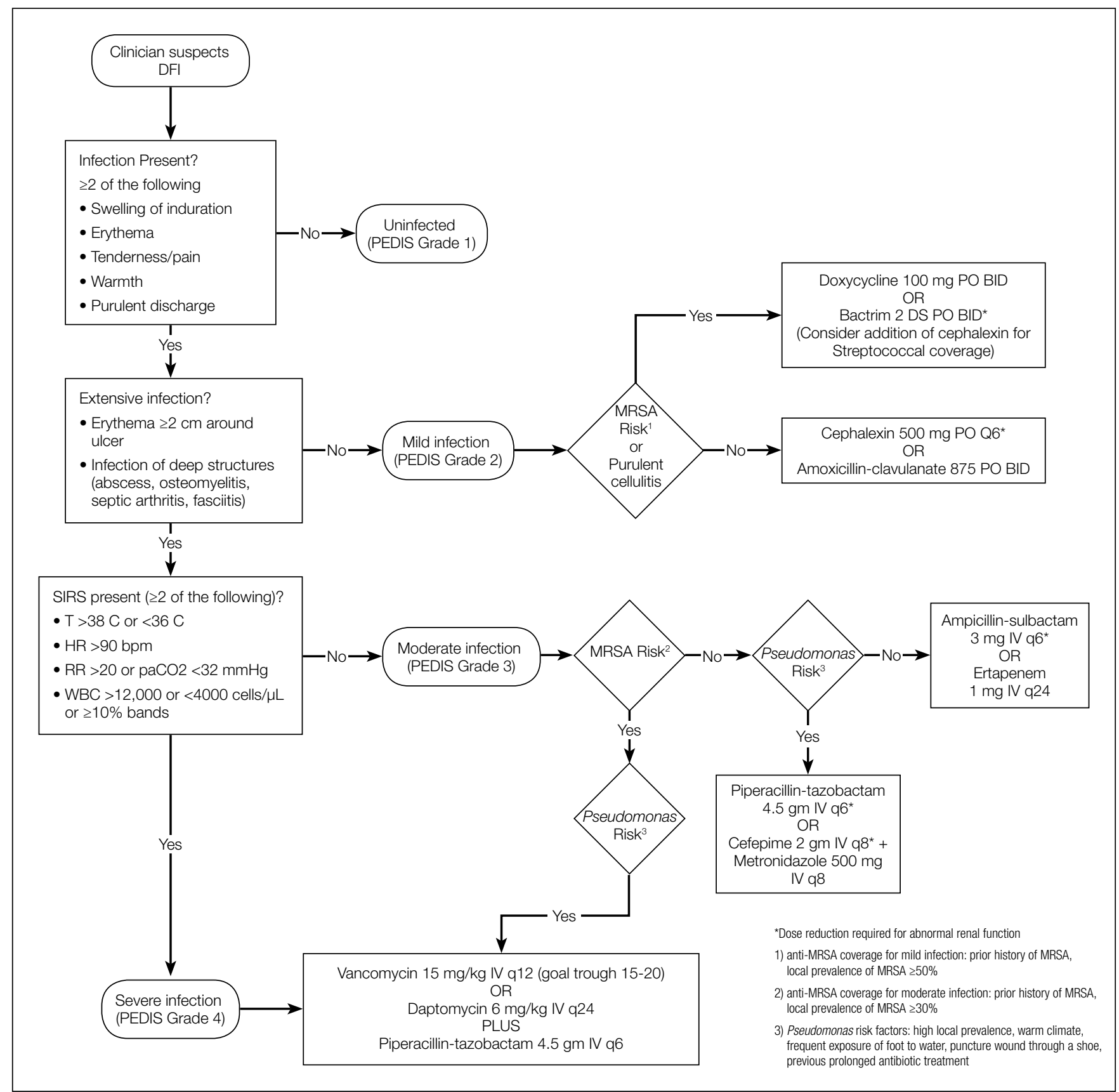

FIG. Algorithm overview of classification and initial treatment of diabetic foot infections.

residence in a chronic care facility, previous amputation, or a high local prevalence of MRSA (50\% MRSA prevalence for mild infections or $30 \%$ prevalence for moderate infections). ${ }^{1,15}$ Fluoroquinolones are no longer effective against S. aureus in most of the United States and should not be used as monotherapy if MRSA is suspected..$^{37,38} \mathrm{~A}$ recent retrospective observational study found that ceftaroline fosamil treatment of DFI was associated with an $81 \%$ success rate, including for patients with comorbidities, MRSA, mixed infections, or surgical intervention, but it has not yet been studied in a comparative trial..$^{39}$ Antipseudomonal therapy is not necessary in most moderate cases and should be re- served for patients who have severe infections (PEDIS grade 4) or specific risk-factors for Pseudomonas. ${ }^{1,15}$ Severe infections, gangrenous wounds, or necrotizing infections require parenteral agents to cover MRSA (ie, vancomycin or daptomycin), Pseudomonas (ie, cefepime or piperacillin-tazobactam), and anaerobes. ${ }^{1,15}$ Anaerobic coverage must be added to cefepime but is not necessary with piperacillin-tazobactam or meropenem. ${ }^{40}$ Definitive therapy should be based on culture results, sensitivity testing, and the patient's clinical response to the empiric regimen. ${ }^{15}$

The duration of antibiotic treatment for DFI is based on the severity of infection and response to treatment (Supplemen- 
TABLE 2. Medical Versus Surgical Considerations for Diabetic Foot Infections

\begin{tabular}{|c|c|}
\hline Factors Favoring Medical Therapy & Factors Favoring Surgical Intervention \\
\hline \multicolumn{2}{|c|}{ Patient Factors } \\
\hline Medically unstable for surgery & High risk for antibiotic-adverse event \\
\hline No contraindications to prolonged antibiotics (ie, recurrent Clostridium difficile) & Intolerance to antibiotics \\
\hline Strong preference to avoid surgery & Preference to avoid long-term antibiotics \\
\hline Good foot perfusion & Poor foot perfusion and penetration of antibiotics \\
\hline Ambulatory patient & Already nonambulatory patient \\
\hline & Indwelling prosthesis vulnerable to metastatic infection (ie, heart valve) \\
\hline \multicolumn{2}{|c|}{ Severity of Infection } \\
\hline \multirow[t]{5}{*}{ Sepsis and soft tissue infection controlled with antibiotics } & Persistent sepsis or spreading infection despite antibiotics \\
\hline & Soft tissue abscess \\
\hline & Compartment syndrome \\
\hline & Necrotizing infection \\
\hline & Pathogen resistant to available antibiotics \\
\hline \multicolumn{2}{|c|}{ Tissue Quality } \\
\hline Minimal tissue destruction, good chance of functional foot with antibiotics alone & Extensive bone or soft tissue necrosis \\
\hline & Exposed or infected joint \\
\hline & Nonsalvageable foot \\
\hline & Visible, chronically exposed trabecular bone in forefoot ulcer \\
\hline
\end{tabular}

tary Table 3). Treatment should continue until the signs and symptoms of infection resolve, but there is no strong evidence to support treatment through complete healing. Healing will usually occur in 1 to 2 weeks for mild infections and in 2 to 3 weeks for moderate or severe infections. However, prescribing antibiotics for a fixed duration is not recommended and can result in an inadequate or unnecessarily prolonged course, with the potential for increased costs, adverse events, and antibiotic resistance. ${ }^{1,15,16}$ Therapy may be shortened by debridement, resection, or amputation, or lengthened in patients who are immunocompromised; have deep, large, necrotic, or poorly perfused wounds; do not undergo resection; or have an implanted foreign body at the infection site. ${ }^{1}$ If the patient does not improve despite targeted antibiotic treatment, providers should assess the need to revascularize, repeat debridement for new cultures, resect any progression of infection, or modify the antibiotic regimen to maximize tissue penetration and minimize drug interactions. ${ }^{1}$

Traditional management of diabetic foot osteomyelitis has relied almost exclusively on resection of all infected bone. However, data have emerged over the last 10 years to support initial medical management of select patients. Further research regarding the optimal treatment regimen and duration is ongoing, with 1 recent, randomized control trial comparing 6 versus 12 weeks of antibiotics for patients treated medically for osteomyelitis finding no difference in remission rates. ${ }^{1,41}$ Patients managed surgically for osteomyelitis are often treated parenterally for at least 4 weeks, but this practice is not based on strong evidence, and guidelines suggest most patients could be switched to highly bioavailable oral agents after a shorter course of intravenous therapy. ${ }^{1,15}$ Guidelines recommend 2 to 5 days of antibiotics after complete resection of infected bone and soft tissue (Supplementary Table 3 ). If the infected soft tissue remains, 1 to 3 weeks of therapy is usually sufficient, while 4 to 6 weeks is often needed if there is residually infected but viable bone. ${ }^{15}$

\section{SURGICAL MANAGEMENT}

Inpatient providers should be familiar with the indications for surgery in DFI patients in order to effectively utilize surgical consultants and ensure critical procedures are completed prior to discharge. Surgical consultation, preferably with a surgeon skilled in foot preservation, is recommended for patients with moderate or severe infections. ${ }^{1,15,33}$ Surgical indications include abscess, necrosis, compartment syndrome, refractory sepsis despite antibiotics, and extensive bone or joint destruction underlying the open wound, as well as other conditions listed in Table 2. While debridement often aids wound healing, it should be avoided in cases with dry eschar, especially when ischemia is present, as the infection will usually resolve with autoamputation.,42,43

In patients with osteomyelitis, the decision between medical and surgical management is complex. Absolute indications for surgical resection include systemic toxicity with associated tissue infection, an open or infected joint space, and patients with prosthetic heart valves. ${ }^{27}$ However, the need for surgery is unclear beyond these absolute indications, and approximately two-thirds of osteomyelitis cases may be arrested or cured with antibiotic therapy alone. ${ }^{1}$ A prospective randomized comparative trial of patients with diabetic foot osteomyelitis found that patients treated with 90 days of antibiotics had similar healing rates, times to healing, and short-term complications as compared with those who underwent conservative bone resection. ${ }^{44}$ While further research is needed to determine which types of patients with osteomyelitis may be successfully treated without surgery, the IWGDF, the IDSA, and osteomyelitis experts have offered guidance on this decision (Table 2).1,15,27 If resection is necessary, hospitalists should request at least 4 specimens to help guide postoperative antibiotic therapy (1 sample for histology and 1 for microbiology, at both the grossly abnormal bone and the bone margin), as negative margin cultures predict a lower relapse risk for infection. ${ }^{1,35}$ 
Every effort should be made to preserve the limb, and urgent amputation is rarely needed except in cases with extensive necrosis or life-threatening infection. Elective amputation may be considered for patients who have recurrent ulceration or irreversible loss of foot function or who would require an excessively prolonged or intensive hospital stay. ${ }^{15}$ All patients with plantar ulcers that are unresponsive to conservative management and limited ankle dorsiflexion should be evaluated for pressure-relieving surgeries, such as Achilles lengthening and gastrocnemius recession. ${ }^{45,46}$ Studies suggest that pressure-relieving surgeries can increase rates of ulcer healing from $88 \%$ to $100 \%$ when added to total contact casting. ${ }^{47}$

\section{CRITERIA FOR DISCHARGE}

Guidelines suggest that patients be clinically stable before discharge, complete any urgent surgery, achieve acceptable glycemic control, and be presented with a comprehensive outpatient plan, including antibiotic therapy, offloading, wound care instructions, and outpatient follow-up (Supplementary Table 4). Physicians must consider patient and family preferences, expected adherence to therapy, availability of home support, and payer and cost issues when creating the discharge plan. ${ }^{15}$

\section{INTERPROFESSIONAL OUTPATIENT CARE}

An effective outpatient care team is critical to ensure wound healing and infection resolution. Efforts should be made to discharge patients to a comprehensive outpatient interprofessional foot care team, with a plan that includes profes-

\section{References}

1. Lipsky BA, Aragón-Sánchez J, Diggle M, et al. IWGDF guidance on the diagnosis and management of foot infections in persons with diabetes. Diabetes Metab Res Rev. 2016;32 Suppl 1:45-74.

2. Hicks CW, Selvarajah S, Mathioudakis N, et al. Burden of infected diabetic foot ulcers on hospital admissions and costs. Ann Vasc Surg. 2016;33:149-158.

3. Number (in thousands) of hospital discharges with peripheral arterial disease (PAD), ulcer/inflammation/infection (ULCER), or neuropathy as first-listed diagnosis and diabetes as any-listed diagnosis United States, 1988-2007. Centers for Disease Control and Prevention website. http://www.cdc.gov/diabetes/statistics/ hosplea/diabetes_complications/fig1_number.htm. Updated 2014. Accessed September 23, 2016.

4. Wilbek TE, Jansen RB, Jørgensen B, Svendsen OL. The diabetic foot in a multidisciplinary team setting. Number of amputations below ankle level and mortality. Exp Clin Endocrinol Diabetes. 2016;124(9):535-540.

5. Jupiter DC, Thorud JC, Buckley CJ, Shibuya N. The impact of foot ulceration and amputation on mortality in diabetic patients. I: From ulceration to death, a systematic review. Int Wound J. 2016;13(5):892-903.

6. Young MJ, McCardle JE, Randall LE, Barclay JI. Improved survival of diabetic foot ulcer patients 1995-2008: Possible impact of aggressive cardiovascular risk management. Diabetes Care. 2008;31(11):2143-2147.

7. Troisi N, Baggiore C, Landini G, Michelagnoli S. How daily practice changed in an urban area after establishing a multidisciplinary diabetic foot program. J Diabe tes. 2016;8(4):594-595.

8. Wang C, Mai L, Yang C, et al. Reducing major lower extremity amputations after the introduction of a multidisciplinary team in patient with diabetes foot ulcer. BMC Endocr Disord. 2016;16(1):38.

9. Rubio JA, Aragón-Sánchez J, Jiménez S, et al. Reducing major lower extremity amputations after the introduction of a multidisciplinary team for the diabetic foot. Int J Low Extrem Wounds. 2014;13(1):22-26.

10. Yesil S, Akinci B, Bayraktar F, et al. Reduction of major amputations after starting a multidisciplinary diabetic foot care team: Single centre experience from Turkey. Exp Clin Endocrinol Diabetes. 2009;117(7):345-349.

11. Dargis V, Pantelejeva O, Jonushaite A, Vileikyte L, Boulton AJ. Benefits of a mul sional foot care, patient education, and adequate footwear. ${ }^{48}$ Team composition varies but often includes representatives from vascular surgery, podiatry, orthotics, wound care, endocrinology, orthopedics, physical therapy and rehabilitation, infectious disease, and dermatology. ${ }^{11-13}$ Interprofessional outpatient clinics can ease the burden of transportation and shorten the time to needed interventions in the case of treatment failure. Follow-up appointments within 1 to 2 weeks postdischarge have been found to reduce the risk of readmission in other high-risk conditions, and this is a reasonable time frame for DFI as well. ${ }^{49}$

\section{CONCLUSION}

DFIs are a common cause of morbidity in patients with diabetes and result in significant costs to the US healthcare system. Hospitalized patients with a DFI require appropriate classification of wound severity and assessment of vascular status, protective sensation, and potential osteomyelitis. Inpatient management of these patients includes obtaining necessary cultures, choosing an antibiotic regimen based on infection severity and the likely causative agent, and evaluating the need for surgical intervention. Prior to discharge, providers should determine a comprehensive follow-up plan and ensure patient engagement. Finally, interprofessional management has been shown to improve outcomes in DFI and should be adopted in both the inpatient and outpatient settings.

Disclosure: The authors report no conflicts of interest. tidisciplinary approach in the management of recurrent diabetic foot ulceration in Lithuania: A prospective study. Diabetes Care. 1999;22(9):1428-1431.

12. Driver VR, Goodman RA, Fabbi M, French MA, Andersen CA. The impact of a podiatric lead limb preservation team on disease outcomes and risk prediction in the diabetic lower extremity: a retrospective cohort study. J Am Podiatr Med Assoc. 2010;100(4):235-241.

13. Hamonet J, Verdié-Kessler C, Daviet JC, et al. Evaluation of a multidisciplinary consultation of diabetic foot. Ann Phys Rehabil Med. 2010;53(5):306-318.

14. Prompers L, Huijberts M, Apelqvist J, et al. Delivery of care to diabetic patients with foot ulcers in daily practice: Results of the Eurodiale study, a prospective cohort study. Diabet Med. 2008;25(6):700-707.

15. Lipsky BA, Berendt AR, Cornia PB, et al. 2012 Infectious Diseases Society of America clinical practice guideline for the diagnosis and treatment of diabetic foot infections. Clin Infect Dis. 2012;54(12):e132-e173.

16. Noor S, Khan RU, Ahmad J. Understanding diabetic foot infection and its management. Diabetes Metab Syndr. 2016;11(2):149-156.

17. Hill MN, Feldman HI, Hilton SC, Holechek MJ, Ylitalo M, Benedict GW. Risk of foot complications in long-term diabetic patients with and without ESRD: A preliminary study. ANNA J. 1996;23(4):381-386; discussion 387-388.

18. Mohler ER, III. Peripheral arterial disease: Identification and implications. Arch Intern Med. 2003;163(19):2306-2314.

19. Hingorani A, LaMuraglia GM, Henke P, et al. The management of diabetic foot: A clinical practice guideline by the Society for Vascular Surgery in collaboration with the American Podiatric Medical Association and the Society for Vascular Medicine. J Vasc Surg. 2016;63(2 Suppl):3S-21S.

20. Noor S, Zubair M, Ahmad J. Diabetic foot ulcer--A review on pathophysiology, classification and microbial etiology. Diabetes Metab Syndr. 2015;9(3):192-199.

21. Wukich DK, Hobizal KB, Brooks MM. Severity of diabetic foot infection and rate of limb salvage. Foot Ankle Int. 2013;34(3):351-358.

22. Wukich DK, Hobizal KB, Raspovic KM, Rosario BL. SIRS is valid in discriminating between severe and moderate diabetic foot infections. Diabetes Care. 2013;36(11):3706-3711.

23. Grigoropoulou P, Eleftheriadou I, Jude EB, Tentolouris N. Diabetic foot infections: 
An update in diagnosis and management. Curr Diab Rep. 2017;17(1):3.

24. Glaudemans AW, Uçkay I, Lipsky BA. Challenges in diagnosing infection in the diabetic foot. Diabet Med. 2015;32(6):748-759.

25. Fleischer AE, Didyk AA, Woods JB, Burns SE, Wrobel JS, Armstrong DG. Combined clinical and laboratory testing improves diagnostic accuracy for osteomyelitis in the diabetic foot. J Foot Ankle Surg. 2009;48(1):39-46.

26. Jeffcoate WJ, Lipsky BA. Controversies in diagnosing and managing osteomyelitis of the foot in diabetes. Clin Infect Dis. 2004;39 Suppl 2:S115-S122.

27. Allahabadi S, Haroun KB, Musher DM, Lipsky BA, Barshes NR. Consensus on surgical aspects of managing osteomyelitis in the diabetic foot. Diabet Foot Ankle. 2016;7:30079.

28. La Fontaine J, Bhavan K, Lam K, et al. Comparison between Tc-99m WBC SPECT/CT and MRI for the diagnosis of biopsy-proven diabetic foot osteomyelitis. Wounds. 2016;28(8):271-278.

29. Bembi V, Singh S, Singh P, Aneja GK, Arya TV, Arora R. Prevalence of peripheral arterial disease in a cohort of diabetic patients. South Med J. 2006;99(6):564-569.

30. Marso SP, Hiatt WR. Peripheral arterial disease in patients with diabetes. J Am Coll Cardiol. 2006;47(5):921-929.

31. Hinchliffe RJ, Andros G, Apelqvist J, et al. A systematic review of the effec tiveness of revascularization of the ulcerated foot in patients with diabetes and peripheral arterial disease. Diabetes Metab Res Rev. 2012;28 Suppl 1:179-217.

32. Brownrigg JR, Apelqvist J, Bakker K, Schaper NC, Hinchliffe RJ. Evidence-based management of PAD \& the diabetic foot. Eur J Vasc Endovasc Surg. 2013;45(6):673-681

33. Wukich DK, Armstrong DG, Attinger CE, et al. Inpatient management of diabetic foot disorders: A clinical guide. Diabetes Care. 2013;36(9):2862-2871.

34. Chakraborti C, Le C, Yanofsky A. Sensitivity of superficial cultures in lower extremity wounds. J Hosp Med. 2010;5(7):415-420.

35. Atway S, Nerone VS, Springer KD, Woodruff DM. Rate of residual osteomyelitis after partial foot amputation in diabetic patients: A standardized method for evaluating bone margins with intraoperative culture. J Foot Ankle Surg. 2012;51(6):749-752.

36. Senneville E, Melliez H, Beltrand E, et al. Culture of percutaneous bone biopsy specimens for diagnosis of diabetic foot osteomyelitis: Concordance with ulcer swab cultures. Clin Infect Dis. 2006;42(1):57-62.

37. Liu C, Bayer A, Cosgrove SE, et al. Clinical practice guidelines by the Infectious
Diseases Society of America for the treatment of methicillin-resistant Staphylococcus aureus infections in adults and children. Clin Infect Dis. 2011;52(3):e18-e55.

38. Richter SS, Heilmann KP, Dohrn CL, et al. Activity of ceftaroline and epidemiologic trends in Staphylococcus aureus isolates collected from 43 medical centers in the United States in 2009. Antimicrob Agents Chemother. 2011;55(9):4154-4160.

39. Lipsky BA, Cannon CM, Ramani A, et al. Ceftaroline fosamil for treatment of diabetic foot infections: the CAPTURE study experience. Diabetes Metab Res Rev. 2015;31(4):395-401.

40. Schultz L, Lowe TJ, Srinivasan A, Neilson D, Pugliese G. Economic impact of redundant antimicrobial therapy in US hospitals. Infect Control Hosp Epidemiol. 2014;35(10):1229-1235.

41. Tone A, Nguyen S, Devemy F, et al. Six-week versus twelve-week antibiotic therapy for nonsurgically treated diabetic foot osteomyelitis: A multicenter open-label controlled randomized study. Diabetes Care. 2015;38(2):302-307.

42. Saap LJ, Falanga V. Debridement performance index and its correlation with complete closure of diabetic foot ulcers. Wound Repair Regen. 2002;10(6):354-359.

43. Steed DL, Donohoe D, Webster MW, Lindsley L. Effect of extensive debridement and treatment on the healing of diabetic foot ulcers. Diabetic Ulcer Study Group. J Am Coll Surg. 1996;183(1):61-64.

44. Lázaro-Martínez JL, Aragón-Sánchez J, García-Morales E. Antibiotics versus conservative surgery for treating diabetic foot osteomyelitis: A randomized comparative trial. Diabetes Care. 2014;37(3):789-795.

45. Bus SA, Armstrong DG, van Deursen RW, et al. IWGDF guidance on footwear and offloading interventions to prevent and heal foot ulcers in patients with diabetes. Diabetes Metab Res Rev. 2016;32 Suppl 1:25-36.

46. Cychosz CC, Phisitkul P, Belatti DA, Glazebrook MA, DiGiovanni CW. Gastrocnemius recession for foot and ankle conditions in adults: Evidence-based recommendations. Foot Ankle Surg. 2015;21(2):77-85.

47. Mueller MJ, Sinacore DR, Hastings MK, Strube MJ, Johnson JE. Effect of Achilles tendon lengthening on neuropathic plantar ulcers. A randomized clinical trial. J Bone Joint Surg Am. 2003;85-A(8):1436-1445.

48. Bus SA, van Netten JJ, Lavery LA, et al. IWGDF guidance on the prevention of foot ulcers in at-risk patients with diabetes. Diabetes Metab Res Rev. 2016;32 Suppl 1:16-24.

49. Jackson C, Shahsahebi M, Wedlake T, DuBard CA. Timeliness of outpatient follow-up: an evidence-based approach for planning after hospital discharge. Ann Fam Med. 2015;13(2):115-122. 